\title{
Paper-based Mixed Reality Sketch Augmentation as a Conceptual Design Support Tool
}

\author{
G.J.D. dos Santos, E.M.A.G. van Dijk, D.M. Vyas \\ Human Media Interaction Group, \\ Department of Computer Science, \\ University of Twente
}

\begin{abstract}
This undergraduate student paper explores usage of mixed reality techniques as support tools for conceptual design. A proof-of-concept was developed to illustrate this principle. Using this as an example, a small group of designers was interviewed to determine their views on the use of this technology. These interviews are the main contribution of this paper. Several interesting applications were determined, suggesting possible usage in a wide range of domains. Paperbased sketching, mixed reality and sketch augmentation techniques complement each other, and the combination results in a highly intuitive interface.
\end{abstract}

\section{Keywords}

Mixed reality, sketch based design, sketch augmentation, paper based interface, computer supported design, design tool

\section{INTRODUCTION}

For thousands of years, paper has served as one of our primary forms of communication. Paper is cheap, light, easily carried around and can be viewed from various orientations. In contrast, digital media usually requires displays which tend to be expensive, awkward to carry around and usually has the user positioned directly in front to view it clearly [1]. Despite the fact that usage of digital media allows for a wider range of expressions, we find that current technology rather than replacing paper has increased our use of it [2]. Books, calendars, newspapers and memo's are still mainly read on paper, and chances are that you are reading these words on paper.

Visually oriented designers tend to work in a very rich environment with a wide variety of tools and materials to hand. Computers are little used in the early, most creative stages of designing [3]. Sketching is still very characteristic of these early stages and paper and pencil is still the medium of choice in many design professions. The design process can be described as a visual task, where ideas must be visualized in order to evaluate relationships among them and drawn representations of these ideas are important in order to make spatial inferences [4]. The vagueness seems to play an important part in the creative process, allowing exploration and reinterpretation of ideas [4]. Currently available Computer Aided Design (CAD) systems

(c) The Author 2009.

Published by the British Computer Society usually require structured information, making it difficult to explore incomplete ideas.

In the development of software that assists in the design process, it is important to understand the user to some degree. Since the introduction of the personal computer in the design studio, its influence in conceptual design has been uncertain [5]. Professional design offices, even with young designers still use traditional or analog media as sketches for ideation [6].

Designers who are discussing and brainstorming usually work in a studio surrounded with sketches. Many designers prefer to use paper media in order to communicate concepts and ideas in the early stages of a given project [7, 8, 9]. Currently, new sketches are mainly created directly on paper on the drafting table before developing a digital mock-up model on the computer.

This digital model can be physically expressed through rapid prototyping techniques [10]. This entails the automatic construction of physical objects using solid freeform fabrication. The most common purpose of rapid prototyping remains the production of prototypes or scale models [11]. The models, both virtual and physical, lead to new ideas, new sketches and eventually this refining process leads to a final product. Even before an external representation, cognitive structures concerning mental images assist the designer to begin conception [12]. Blinn [13] postulates that the creative process has two phases: firstly, moving from chaos to order and secondly, from ideation to implementation. Most computerbased design tools are primarily focused on the second phase, and there is limited support for digital tools where people can play with ideas in a freeform manner.

For novice designers dealing with complex shapes, an intuitive representation is needed in order to understand the concept and overcome design issues [14], and the initial sketches have proven to be difficult to grasp fully. With new technology it may be possible to enhance this process. We propose that a novel combination of mixed reality techniques and digital sketch augmentation would be able to create an interpretation directly from an initial sketch on paper in an intuitive and relatively freeform manner, suitable for supporting conceptual design. We developed a working prototype that enhances and supports paper-based sketching in the early, conceptual stages of the design process. This prototype was then used as an illustration to explore how such a system may improve upon current practices and in what ways it could contribute to or otherwise enhance the design process.

In this paper we first present a prototype that combines augmented reality with sketch inferring techniques. This program mimics the process of moving from paper sketch to scale model and its main purpose is to serve as a proof-ofconcept for mixed reality sketch augmentation. We then explore how such a system could aid in the design process by having discussions with several designers after the prototype was 
demonstrated to them. In these discussions we identify various applications and suitable domains for the combination of sketch augmentation and mixed reality techniques. Although the prototype's workings will be described in detail, the main focus of this research lies with the designers' opinion on how paperbased augmented reality technology could be useful to them.

Our findings were encouraging and we suggest that there may be applications in other domains such as art and entertainment.

\section{RELATED WORK}

There have been several research projects that have investigated various means of linking paper to digital media in an attempt to combine the advantages of both approaches $[15,16]$. The Digital Desk $[15,17]$ is a real physical desk, which projects electronic images down onto the desk and upon paper documents and responds to interaction with pens or bare fingers. The digital desk is also able to read paper documents placed on the desk and create electronic copies.

Researchers such as Ishii [18] have explored the use of tangible object interfaces for tabletop collaboration. Regenbrecht extends the idea of tangible user interfaces and demonstrates the benefits in a novel video conference system [19].

Using sketches as a basis for 3D models has also been researched, and is even commercially available. In [20] 3D models are inferred directly from a sketch, as a more intuitive modeling tool for non-expert users. Sketching interfaces for freeform models are explored in [21], which resulted in Teddy, a real-time java application that constructs plausible 3D polygonal surfaces from a $2 \mathrm{D}$ silhouette. Teddy was designed to work with a stylus and is controlled with gestures.

The modeling tool Shapeshop [22] uses procedural implicit surface techniques to quickly assemble 3D models from sketches. As is the case with [20] and [21], the sketches are fully digital.

Solutions proposed to integrate the sketch in the digital design process exist in various forms as well. The Sketchpad project [23] serves indirectly as the inspiration for most modern CAD software. Another approach is digitally imitating a real sketch [24] or using sketches as command triggers because of their intuitive characteristics [25].

\section{AUGMENTED RACETRACK DESIGN}

When discussing a new technology a lot of imagination is needed in order to discuss its various aspects. By demonstrating a prototype the technology becomes less abstract, and more informed opinions can be expressed. For this reason we have developed a proof-of-concept that illustrates various concepts and possibilities of mixed reality sketch augmentation.

The key points of interest that were chosen include intuitive usage, 3D interpretation, freeform sketching, real-time performance and animation. These concepts highlight various strong points in both paper-based sketching and virtual modeling.

In contrast to the systems mentioned in section 2, our prototype seeks to support conceptual sketching through mixed reality with a purely physical interface.

\subsection{Hardware composition}

As one of the goals of the prototype was accessibility, some effort was made to avoid exotic hardware of any kind. We postulate that familiarity with the hardware involved reduces the perceived complexity of the operating procedures.
The equipment required for augmented reality applications generally includes a camera, a display method and a computer. The development system consisted of a common webcam and a laptop computer. The webcams quality is important for the program, where lower resolutions decrease the fidelity of the final model and lower frame rates have proven to be detrimental to the user experience. The webcams frame rate is a bottleneck in the programs and may result in slower than real-time behavior that negatively impacts users' experience. Another notable issue is the amount of noise in the image, as this directly influences the frame-to-frame stability and precision of the generated model. The final augmented video stream is shown on the laptop screen.

\subsection{Software Architecture}

Three public libraries were selected as a basis for the Augmented Reality eXperimental (ARX) program. Augmented Reality techniques were adapted from the ARToolkit [26], with additional image processing done with openCV [27]. Finally, the resulting scene was composited and displayed with the openscenegraph (OSG) library [28]. From a high level perspective there are two phases in the program: processing the video data, and generating the various models.

Firstly, the program captures a video frame and attempts to locate a square with a thick black border, presumably on a piece of paper. If a square is found in the video image, it is distorted through some geometric transformation dependant on the physical position and orientation of the square relative to the camera. This transformation can be determined and used to normalize the image. We will elaborate on this in section 3.3. After normalizing the image, the pattern within the square is analyzed and a 3D model is generated. The 3D model is further refined in order to determine a path, which is used to guide an animated model. This process will be detailed in section 3.4.

\subsection{Paper tracking}

For determining the position and orientation of the target sketch to the camera we make use of the ARToolkit library, which is a relatively simple marker-based optical tracking system, and allows virtual imagery to be superimposed upon live video of the real world. The library is designed to work with webcams or video cameras and with uniquely identifiable fiduciary markers.

Originally the process includes symbol recognition, but in our ARX program this aspect of the process from ARToolkit has been replaced by image analysis and model generation code. From the original process both the marker detection and the transformation calculation have been preserved.

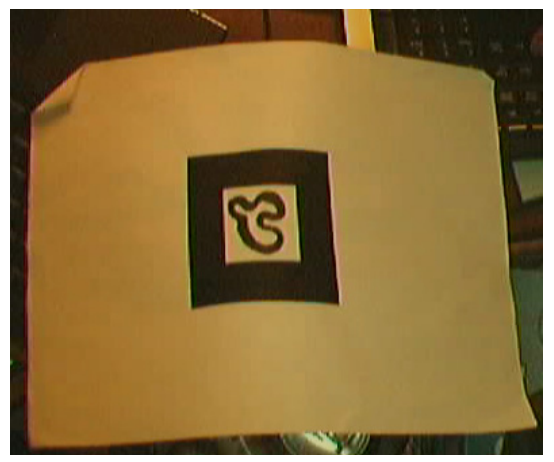

Figure 1 - Fiduciary marker with a racetrack sketch

The camera captures a video image of the real world and sends it to the computer. This image is then converted to black and white and searched for any black square shapes. This a typical description of a fiduciary marker [Fig.1] commonly used in 
marker-based augmented reality applications [29, 30]. If such a fiduciary square is found, the position and orientation of the square relative to the camera is calculated. The library then proceeds to normalize the contents of the square to a fixed resolution and orientation [Fig.2]. In the original application this is then used to match against a library of known symbols, but in our system it is the basis upon which a model is generated.

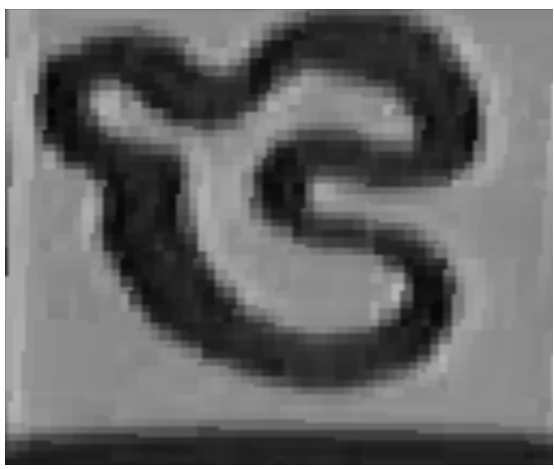

Figure 2 - Normalized sketch

\subsection{Model generation}

The basic image format consists of color values per pixel. The tracking process normalizes the image resolution and orientation, which ensures that the image is stable when compared to the previous video frame. In our system multiple models are generated from this image, varying slightly in the level of processing needed.

The first model which is generated is a heightfield [Fig.3], which interprets the color of each pixel as a height value for a regular grid.

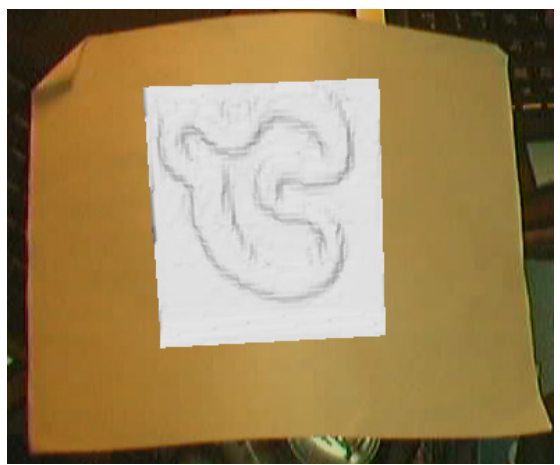

Figure 3 - Generated 3D heightfield

The second model which is generated is the road, which is placed on top of the heightfield. This model is acquired by contour analysis of the image [Fig.4], which should result in two contours - the inner and outer edges of any lines drawn. These lists of points usually resemble each other in shape, but the number of points in both lists varies greatly. In order to create a visible road a simple method was devised to create a triangle strip. For this algorithm, it is assumed that exactly two contours are found in the contour analysis, and if more than two were found only the first two are used in the model generation. These lists are compared to one another and for each point from the outer contour the nearest point in the inner contour is found. These connections form a strip which can be drawn.

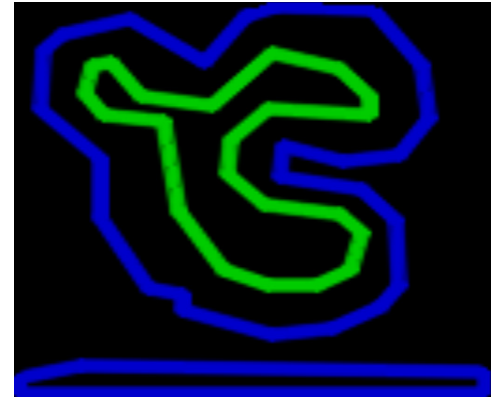

Figure 4 - Contours found in the image

This approach places several restrictions on the drawing, such as requiring the line to be thick enough to result in two contours. Another limitation is that the line must form a loop and may not cross. Although these are not severe limitations in the specific case of racetrack design, the freeform nature of sketching is impeded because of this. Ignoring the aforementioned limitations of the ARX program tends to result in slightly unexpected behavior.

The third and last generated model concerns the path which the car should follow. This model is based on the road model, and approximates the central line found in the road [Fig.5]. The path is constructed from the road model, by taking the midpoints between each pair of vertices.

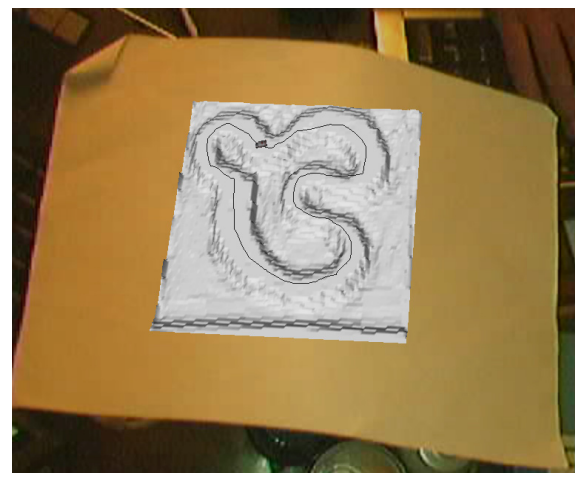

Figure 5 - Path on top of model

The car position and orientation is determined by finding the closest point on the path to the cars' current position, determining the next point in the path and steering the car towards this next point.

At the final stage in the image assembly all the generated models are combined and textured [Fig.6]. The position and orientation of the paper in relation to the camera, which was determined at an earlier stage, is now applied to the entire generated model, giving the illusion that the model sticks on top of the paper.

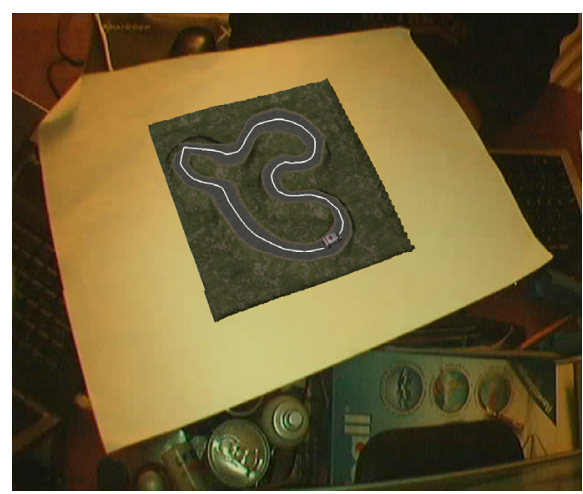

Figure 6 - Completed model 


\section{DESIGNER FEEDBACK}

After the prototype was completed we performed a small-scale user research. We focus on using the combination of mixed reality techniques and sketch augmentation as a design support tool, and as such we have conducted several interviews with the prospective users of such a tool. We will first describe the subject group and the structure of the interview, followed by short descriptions and rationale of each question asked.

\subsection{User group}

The prospective users are mainly designers, and as such we have interviewed mainly industrial design undergraduates and researchers. A total of 9 interviews were conducted, all of which were conducted at the University of Twente, but the individual specializations and aptitudes of the interviewees were relatively varied. The interviews included both men and women, and ages ranged from around 20 to around 50 years old. All of the subjects were familiar with both CAD applications and rapid prototyping, and some had experience in digital sketching.

\subsection{Interview structure}

The interviews started with a short introduction on what the research is about and what the ARX program does. After this introduction, the prototype program is presented as a proof-ofconcept for mixed reality sketch augmentation and the interviewee is provided with an opportunity to try it out for him or herself. Afterwards, a list of five thematic questions is discussed. The questions are expected to provoke a discussion in which interesting opinions arise. In some cases the questions were supplemented with follow-up examples, clarifications or slight variations on the original question.

The first theme focuses on the concept of using paper as an input device, the second attempts to find some benefit to using mixed reality techniques. Third is a comparison with rapid prototyping as a conception tool and fourth is an inquiry as to the perceived usefulness of a tool such as the ARX program in the design process. The fifth and last theme is an opportunity for the designer to voice opinions on altering or improving the program in any way.

\subsection{Paper as an input medium}

We have chosen paper as an input medium with the assumption that it is a very intuitive and convenient medium when attempting to communicate sketched ideas. However, when compared to input devices such as mice and keyboards it becomes clear that paper also has a lot of disadvantages for digital purposes, such as the precision and interpretation aspects. At this point, we ask the designer to form an opinion on using paper directly as an input device for the computer.

Several designers had a personal preference for working with paper instead of digital input devices such as mice or even a tablet. The tactile and immediate visual feedback provided by paper plays an important role in the ideation experience.

Another common response was that paper is a more accessible medium than a mouse or keyboard approach, needing far less experience for effective use. Input devices require a certain amount of knowledge to operate, and in this case these prerequisites have been reduced to a minimum. This would allow a wide range of people to make use of it.

The matter of expressive freedom seems to play a minor role, as this can largely be compensated for with experience. It seems that when one starts to work with a new medium the possibilities seem endless, and only when the user becomes proficient the medium's limitations start to restrict the design experience. When the purpose of the supporting program is to assist in the conception stage, the tools' accuracy does not need to be very high.

\subsection{Mixed reality for design tools}

A second matter which is discussed is the perceived benefit of using mixed reality techniques in design tools. While multiple techniques may perform the integration of the virtual and the real world, we mainly discuss the technique used in the ARX prototype, namely marker-based superimposition of virtual models in a video feed.

The largest advantage of using this technique is that the real world is being used as a context for the design. While the ARX program is too limited to make use of this, one could imagine augmented sketches being used as a preview method. For example, one designer suggested placing a sketch on a curved surface and then seeing the surface covered in the sketch.

Another advantage is that the generated model feels more realistic, and closer to being physical than when viewing it on a screen. The responsiveness of the program forms a sharp contrast with the normal workflow for CAD applications, and was mentioned to be a welcome change from typical computer based design tools.

\subsection{Conventional design tools}

The third question asks the designer to compare the ARX program to another, more conventional design tool - namely $3 \mathrm{D}$ printing and other rapid prototyping techniques. When the intent of using a specific design tool is to clarify or explore concepts the two can be compared in some ways, although the techniques remain very different.

Various differences of the ARX program were noted, such as the dynamic appearance of the prototypes when compared to the static models that are produced by 3D printing. Even though the virtual model can display more varied behavior, it cannot perform all of the roles from physical models. For example, physical models are also used to confirm behavior and shapes, which have been conceived as a sketch or virtual model but remain theoretical until they have been physically created.

\subsection{Usefulness}

It is then that we turn our attention to the usefulness of the proposed technique as a design tool. We ask if this kind of tool would be useful in the design process in general, whether its usefulness is limited to specific domains.

It seems that it is too early to determine a specific use of this technology, but an interesting possibility is to engage a larger group of people in the early stages of the design process. Usually, a textual or verbal description is given on what kind of product is desired, after which designers go through the design process with a limited amount of communication and feedback with the original contractors. The accessibility of this type of technology allows the original contractors to play a larger, more effective role in the conception stage.

Some immediate domains that may benefit from such a tool were identified: city planning, landscaping and interior decoration for example. Three dimensional modeling would require a lot more from the sketch augmentation, although the ARX example of shape extrusion seems useful for detail work on the outer layer of larger models. Adding detail ornaments on an otherwise complete CAD-model is currently a rather timeconsuming task, and because of this it is considered not practical to experiment with various patterns. 
The animations in the prototype seemed useful, for example in visualizing traffic flow. For these types of applications the paper interface is considered inadequate, but a touch screen would provide a reasonable alternative.

Several designers also suggested that a combination of the ARX program with 3D printing or milling would provide an alternate, faster though less accurate workflow and would allow a designer to see his or her ideas become reality in a more swift and intuitive manner. This would allow much more experimentation than is currently feasible in a limited period of time, which can potentially lead to higher quality products.

Another area where this type of program may be useful is in teaching the design process. Currently the industrial design curriculum at the University of Twente contains both sketching on paper and CAD applications, and paper-based sketch augmentation may assist in smoothing the transition between the two.

\subsection{Suggestions}

The last question offers the designer a chance to opinionate improvements or alterations for the ARX program. In the course of the interview many ideas are generated, some of which only require minor adjustments to the program.

Some suggestions we received were the inclusion of more elaborate pattern recognition, most notably symbol recognition. This would allow for more elaborate scene compositions, and would focus more on specific domains. A typical example of an interesting program that makes use of this is the design of electrical circuits. Another suggestion was to port the program to a smart phone, so that the experience becomes more ubiquitous. This could make it simpler for users to become engaged in the design process, for example by drawing on a napkin. One designer suggested focusing on incrementally updating sketches, using computer hardware to save and print out 'backup' sketches. The printing process could be integrated with conventional rapid prototyping, further simplifying the designers' regular workflow.

\section{DISCUSSION}

We have seen many possible applications for the combination of mixed reality and sketch augmentation and we feel that these techniques complement each other and have a positive synergetic relation. That being said, we have only superficially explored this combination and during the interviews it became clear that many aspects from both the individual techniques and the combination warrant a more thorough investigation.

All of the interviewees had a positive attitude with regard to the concept as a whole, but some also noted that this is probably a transitional type of technology - while paper usage has been and still is very widespread, some designers mentioned during the interviews that they believe that some form of digital media might take its place.

\subsection{Further development}

While demonstrating the prototype, many possible improvements were suggested. These suggestions could roughly be separated in improvements to the mixed reality techniques and improvements to the sketch augmentation. As we did not make use of the state of the art of either, these aspects of the program leave a lot to be desired.

One of the main shortcomings of the ARX prototype is the limited drawing space within the fiduciary marker. There are various possible approaches to address this problem, such as separating the marker tracking from the model generation. This approach would use the space next to the marker as a basis for model generation instead of the space inside the marker. Another approach to this problem is to disregard the use of markers and attempt to detect sketches by point of interest tracking.

The techniques and hardware that were used resulted in a somewhat unstable image, which was somewhat sensitive to lighting conditions. We expect that both better hardware and more advanced mixed reality techniques may greatly improve the programs user experience.

A very different aspect of the prototype that can be improved upon is the sketch augmentation process, as the approach we used is neither generic nor efficient. An interesting extension would be to support the interpretation of colors, instead of only black and white.

The interpretation process could conceivably be extended with various optical pattern recognition techniques, such as symbol recognition or edge detection. Most of the future systems proposed by the designers in fact require some of these techniques in order to function properly.

\subsection{Further research}

Other areas that may be of academic interest are possible applications other than as a design tool. This type of technique may be useful for example in the entertainment industry or for art conception or production.

We also feel that the ubiquitous nature of mixed reality sketch augmentation has contributed to its positive reception. The combination of technologies may be compounded further with other 'natural' interface techniques such as speech recognition, in order to provide users with a more complete and intuitive interface that helps to resolve ambiguity in drawings.

\section{CONCLUSION}

We have presented a prototype that demonstrates the principle of paper-based mixed reality sketch augmentation. We conducted several interviews with industrial design students and teachers where we demonstrated the ARX program and explored various aspects of such a tool. We have found that several designers displayed a preference for conceptualizing on paper.

Currently, mixed reality sketch augmentation would mostly be useful in the ideation stage of the design process, but many other possible applications have been identified.

Several interesting suggestions for digitally augmenting the design process through paper-based sketches were also obtained during the interviews, such as adding 'save-and-restore' capabilities to sketching or using it as a direct interface for rapid prototyping or milling systems.

In conclusion, paper-based sketching, mixed reality and sketch augmentation techniques complement each other, and the combination results in a highly intuitive interface that mediates between freehand drawing and computer aided modeling while preserving the context provided by the physical world and the tactile feedback provided by paper.

\section{REFERENCES}

[1] Sellen, A. J. and Harper, R. H. (2003) 'The Myth of the Paperless Office'. MIT Press.

[2] Vanasselt, W. (1998) 'No End to Paperwork' in World Resources 1998-99, pages 1-6 
[3] Hyde, R. (1989) 'Design procedures in architectural design: applications in CAAD', in Design Studies 10 (October 1989), pages 239-245

[4] Ballay, J. (1987) 'An Experimental View of the Design Process', in System Design: Behavioral Perspectives on Designers, tools and Organisations, W. Rouse, K. Boff (Eds.). North-Holland, 1987, pages 65-82

[5] Willey, D. (1999). 'Sketchpad to 2000': From Computer Systems to Digital Environments. Proceedings of the eCAADe Conference, Architectural Computing from Turing to 2000: pages 526-532. Liverpool: Education and research Computer Aided Architectural Design in Europe

[6] Dorta,T., and Pérez, E. (2006), 'Immersive Drafted Virtual Reality: a new approach for ideation within virtual reality', in: Luhan, G.,Anzalone, P., Cabrinha, M. and Clarke, C., eds., Synthetic Landscapes - Digital Exchange, ACADIA Conference, Louisville, pages 392-402

[7] Oxman, R., O. Shaphir, and M. Yukla. (1998). 'Beyond Sketching: Visual reasoning through re-presentation in cognitive design media'. in Proceedings of the CAADRIA'98 Conference: pages 337-346. Osaka: Computer Aided Architectural Design Research in Asia

[8] Gross, M., and E. Y. Do. (1996). 'Ambiguous Intentions: A Paper-Like Interface for Creative Design'. Proceedings of the ACM UIST Conference: pages 183-192. Cambridge: User Interface Software Technology

[9] Garner, S. (2000). 'Is Sketching Still Relevant in Virtual Design Studios?', in Proceedings of the DCNet Conference, pages 1-6. Sydney

[10] Gebhardt, A. (1996), 'Rapid Prototyping', Carl Hanser Publising

[11] Chua, C.K., Leong, K.F., Lim, C.S. (2003) 'Rapid Prototyping: Principles and Applicactions', World Scientific

[12] Bilda, Z. and J. S. Gero. (2005). 'Do We Need CAD during Conceptual Design?'. Proceedings of the CAAD Futures Conference: pages 155-164. Vienna: Computer Aided Architectural Design Futures

[13] J. Blinn (1990), 'The ultimate design tool', IEEE Computer graphics and Applications 10(6), pages 90-92

[14] Do, E. Y. (1996). 'The Right Tool at the Right Time: drawing as an interface to knowledge based design aids', in Proceedings of the ACADIA Conference, Design computation: Collaboration, Reasoning, Pedagogy, Tucson: Association for Computer-Aided Design in Architecture, pages 191-199

[15] Wellner, P. (1993), 'Interacting with paper on the DigitalDesk'. Commun. ACM 36, 7 (Jul. 1993), pages 8796

[16] Luff, P., Heath, C., Norrie, M., Signer, B., and Herdman, P. (2004). 'Only touching the surface: creating affinities between digital content and paper'. In Proceedings of the 2004 ACM Conference on Computer Supported Cooperative Work (Chicago, Illinois, USA, November 6$10,2004)$. CSCW '04. ACM, New York, NY, pages 523532

[17] Newman, W., Wellner, P. (1992) 'A desk supporting computer-based interaction with paper documents', in
Proceedings of the SIGCHI conference on Human Factors in computing systems, (Monterey, California, May 3-7, 1992), pages 587-592

[18] H. Ishii (1997), 'Tangible bits: towards seamless interfaces between people, bits and atoms', in Proceedings of CHI, pages 234-241

[19] H. Regenbrecht (2004), 'Using augmented virtuality for remote collaboration'. Presence: Teleoper. Virtual Environments 13(3) pages 338-354

[20] Levet, F. and Granier, X. (2007), 'Improved skeleton Extraction and surface generation for sketch-based modeling', in Proceedings of Graphics Interface 2007 (Montreal, Canada, May 28-30, 2007). GI'07 volume 234, ACM, New York, NY, pages 27-33

[21] Igarashi, T., Matsuoka, S., and Tanaka, H. (2007), 'Teddy: a sketching interface for 3D freeform design', in $A C M$ in SIGGRAPH 2007 Courses, (San Diego, California, August 5-9, 2007), SIGGRAPH'07, ACM, New York, NY, page 21

[22] Wyvill, B., Foster, K., Jepp, P., Schmidt, R., Sousa, M.C., Jorge, J.A. (2005), 'Sketch Based Construction and Rendering of Implicit Models', In Proceedings of the first Eurographics Workshop on Computational Aesthetics in Graphics, Visualization and Imaging 2005, (Girona, Spain, May 18-20, 2005), Eurographics Association, pages 67-74

[23] Sutherland, I. (1963). 'Sketchpad: a man-machine graphical communication system'. In Seminal Graphics: Pioneering Efforts That Shaped The Field, ed. R. Wolfe, ACM Siggraph 98, pages 391-408

[24] Jatupoj, P. (2005). 'Sketchboard: the simple 3D from architectural sketch recognition'. Proceedings of the CAADRIA'05 Conference: pages 3-22. New Delhi: Computer Aided Architectural Design Research in Asia

[25] Brito, T., M. J. Fonseca, and J. Jorge. (2005), 'DecoSketch: Towards Calligraphic Approaches to Interior Design'. Proceedings of the eCAADe Conference, Digital Design: The Quest for New Paradigms: pages 665-670, Lisbon: Education and research Computer Aided Architectural Design in Europe

[26] ARToolKit, available at http://www.hitl.washington. edu/artoolkit/ (accessed Januari 9th, 2009)

[27] OpenCV, available at http://sourceforge.net/project/ showfiles.php?group id $=22870$ (accessed Januari 9th, 2009)

[28] openSceneGraph, available at http://www.openscenegraph. org (accessed Januari 9th, 2009)

[29] Fiala, M. (2005) 'Artag, A Fiducial Marker System using Digital Techiques', in IEEE Proceedings on Computer Vision and Pattern Recognition (San Diego, June, 2005), CVPR'05, Volume 2, IEEE Computer Society, pages 590596

[30] Kato, H., Billinghurst, M. (1999), 'Marker Tracking and HMD Calibration for a video-based Augmented Reality Conferencing System', in Proceedings of the 2nd International Workshop on Augmented Reality, (San Fransisco, California, October 20-21, 1999), IWAR'99, pages $85-94$ 